\title{
Incretin-based therapies: where will we be 50 years from now?
}

\author{
Juris J. Meier ${ }^{1} \cdot$ Michael A. Nauck ${ }^{1}$
}

Received: 4 February 2015 / Accepted: 17 March 2015 / Published online: 21 May 2015

(C) Springer-Verlag Berlin Heidelberg 2015

\begin{abstract}
The development of incretin-based therapies (glucagon-like peptide 1 [GLP-1] receptor agonists and dipeptidyl peptidase-4 [DPP-4] inhibitors) has changed the landscape of type 2 diabetes management over the past decade. Current developments include longer-acting GLP-1 receptor agonists, fixed-ratio combinations of GLP-1 analogues and basal insulin, as well as implantable osmotic minipumps for long-term delivery of GLP-1 receptor agonists. In longer terms, oral or inhaled GLP-1 analogues may become a reality. In addition, oral enhancers of GLP-1 secretion (e.g. via G-protein-coupled receptors, nuclear farnesoid-receptor X and the G-proteincoupled bile acid-activated receptor [TGR5]) are currently being explored in experimental studies. Combination of GLP-1 with other gut hormones (e.g. peptide YY, glucagon, gastrin, glucose-dependent insulinotropic polypeptide [GIP], secretin, cholecystokinin, vasoactive intestinal polypeptide and pituitary adenylate cyclase-activating polypeptide) may enhance the glucose- and weight-lowering effect of GLP-1 alone, and dual or triple hormone receptor agonists may even exploit the properties of different peptides with just one molecule. There is also an increasing interest in employing incretin-based therapies in other areas, such as type 1 diabetes, impaired glucose metabolism, obesity, polycystic ovary syndrome, non-alcoholic fatty liver disease (NAFLD)/non-alcoholic steatohepatitis (NASH), psoriasis or even neurodegeneration. Thus, incretin-based therapies may continue to broaden the therapeutic spectrum for type 2 diabetes and for various
\end{abstract}

Juris J. Meier

juris.meier@rub.de

1 Division of Diabetes and GI Endocrinology, University Hospital St Josef-Hospital, Ruhr-University Bochum, Gudrunstr. 56, 44791 Bochum, Germany other indications in the coming years. This is one of a series of commentaries under the banner ' 50 years forward', giving personal opinions on future perspectives in diabetes, to celebrate the 50th anniversary of Diabetologia (1965-2015).

Keywords GIP · GLP-1 · Gut hormones · Type 2 diabetes
Abbreviations
DPP-4 Dipeptidyl peptidase-4
GIP Glucose-dependent insulinotropic polypeptide
GLP-1 Glucagon-like peptide-1
IFG Impaired fasting glucose
IGT Impaired glucose tolerance
PYY Peptide YY

\section{Where are we today and where have we come from?}

The development of incretin-based therapies is a splendid and unique example of the successful development of novel glucose-lowering medications based on basic research findings from academic researchers. Physiological experiments in human volunteers revealed different increments in insulin secretion after oral and intravenous glucose administration, which were subsequently attributed to the actions of the incretin hormones glucose-dependent insulinotropic polypeptide (GIP) and glucagon-like peptide-1 (GLP-1) [1]. These peptide hormones were later synthesised and prepared for intravenous infusion in patients with type 2 diabetes. On this basis, the glucose-lowering activity of GLP-1 was first discovered by academic researchers. Later on, dipeptidyl peptidase- 4 (DPP-4) inhibition was identified as an effective way of raising endogenous GLP-1 levels [2]. These clinical research findings have prompted the development of GLP-1 receptor 
agonists and DPP-4 inhibitors [3]. Today, a variety of DPP-4 inhibitors are available. Despite structural and pharmacokinetic differences, the pharmacodynamic properties and clinical efficacy of these compounds are largely comparable. Various GLP-1 analogues have been developed using different modes of protraction (e.g. amino acid exchange, fatty acid side chains, covalent coupling to larger molecules, depot preparation based on microspheres). As a general rule, these compounds can be categorised as short-acting (predominant activity on post-meal glycaemic rises) and long-acting GLP-1 analogues (24 $\mathrm{h}$ active with predominant effect on fasting glucose and somewhat reduced effectiveness on post-meal glycaemic excursions) [4]. The different individual characteristics of the various GLP-1 analogues and the DPP-4 inhibitors have already allowed for some individualisation of care within the class of incretin-based therapies.

\section{Future developments already visible}

Recent developments in the field of GLP-1 receptor agonists have largely focused on longer-acting preparations (i.e. injection once weekly or even less frequently) and more convenient delivery systems or pen devices. A number of such onceweekly GLP-1 receptor agonists have already been introduced or are currently undergoing clinical trials [4]. In light of the narrow therapeutic window defined by the balance between glucose-lowering efficacy and (so-called) gastrointestinal side effects, it seems unlikely that future long-acting GLP-1 receptor agonists will provide substantially greater efficacy without inducing more side effects compared with the already available compounds.

Alternative developments are already on the way. One of them is an osmotic minipump that releases exenatide continuously for $\sim 6$ months or even longer after implantation into subcutaneous tissue (Fig. 1). Furthermore, fixed-ratio combinations delivering GLP-1 analogues and basal insulin at the same time have just become available in some countries. Advantages of such fixed-ratio combinations include overall better $\mathrm{HbA}_{1 \mathrm{c}}$ control compared with the individual compounds and less frequent nausea than observed with GLP-1 receptor agonist monotherapy [5].

In contrast to the trend of developing longer-acting compounds/preparations, it may also be of interest to further explore the therapeutic potential of even shorter-acting GLP-1 analogues for prandial glucose control. Thus, whereas the currently available short-acting GLP-1 analogues have plasma half-lives of $\sim 3-4 \mathrm{~h}$, future compounds exhibiting half-lives of 1-2 $\mathrm{h}$ may exploit the mechanism of delaying gastric emptying even more specifically. Such compounds could potentially complement the therapy with long-acting GLP-1 receptor agonists, analogous to a basal-bolus insulin therapy. A downside of even shorter-acting GLP-1 analogues might be a
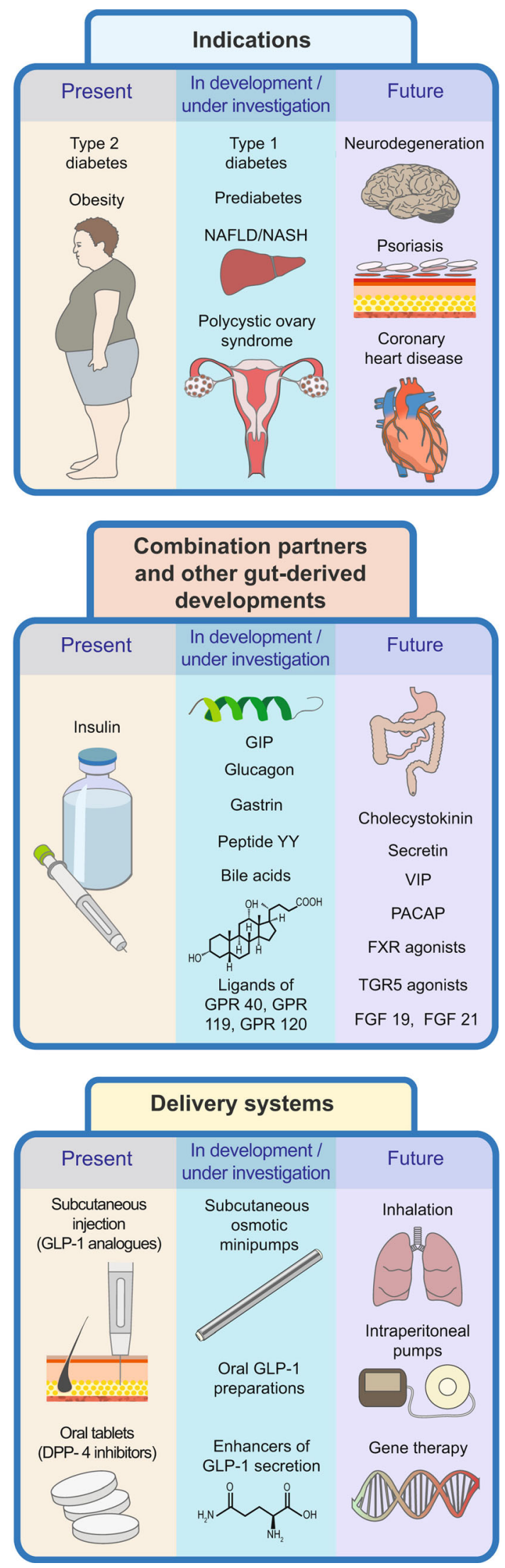
4 Fig. 1 The present state of the art (red; left fields), current clinical developments (blue; middle fields) and the potential future (green; right fields) of incretin-based therapies in terms of delivery systems, combination partners and other gut-derived developments, and therapeutic indications. FGF, fibroblast growth factor; FXR, farnesoidreceptor X; GPR, G-protein-coupled receptor; NAFLD, non-alcoholic fatty liver disease; NASH, non-alcoholic steatohepatitis; PACAP, pituitary adenylate cyclase-activating polypeptide; TGR5, G-proteincoupled bile acid-activated receptor; VIP, vasoactive intestinal polypeptide

higher incidence of nausea and vomiting. DPP-4 inhibitors are being developed for administration once weekly, too.

\section{Future developments not yet visible}

\section{New ways of delivering incretin-based drugs}

Intravenously administered GLP-1 has consistently been shown to fully normalise glycaemia [3]. In contrast, the subcutaneously administered GLP-1 analogues have provided strong glucose-lowering efficacy, but still fail to fully normalise glycaemia in a substantial proportion of patients. The percentage of patients achieving $\mathrm{HbA}_{1 \mathrm{c}}$ levels below $6.5 \%$ ranges from $\sim 25-50 \%$ with DPP-4 inhibitors and from $\sim 40-70 \%$ with GLP-1 receptor agonists [6]. It is also noteworthy that nausea typically limits further dose escalation with subcutaneous GLP-1, but rarely occurs during intravenous GLP-1 infusion. These observations have suggested that delivery routes other than subcutaneous injection might be favourable [7]. Although proteolytic degradation by gastric acid limits the oral administration of GLP-1, chemically modified formulations of GLP-1 suitable for oral administration are likely to be available in 50 years. Inhalation may be another option, but local proliferative effects of GLP-1 in the lungs may limit such an approach. Other routes, such as buccal or rectal administration, have been explored in the past, but are unlikely to play a role in clinical practice. Other routes of delivering GLP-1 directly into the systemic circulation (e.g. intraperitoneally, intravenously) may need to be further explored in the future, especially since - other than with insulin - only a relatively constant level of a GLP-1 receptor agonist needs to be maintained, high enough to be effective and low enough not to elicit adverse reactions (Fig. 1).

Enhancing endogenous GLP-1 secretion is another promising future strategy. Current strategies to target G-proteincoupled receptors (GPR 40, GPR 119 and GPR 120) on intestinal L cells have shown theoretical potential for diabetes therapy, but the compounds that were effective were not safe enough [8]. Alternative approaches include nutrient pre-loads, predominantly based on protein components. The fact that endogenous GLP- 1 concentrations increase by $\sim 5-10$-fold after bariatric surgery [9] strongly underlines the theoretical potential for enhancing GLP-1 secretion, even though an ideal strategy to stimulate L cell secretion has yet to be discovered.

It is also conceivable that gene therapy may reach a level that allows for a broad clinical application in 50 years from now. In this regard, genetic (e.g. adenoviral) transfer of the proglucagon gene to other cell types might lead to enhanced secretion of GLP-1 from endogenous sources (Fig. 1).

\section{GIP receptor agonists/antagonists}

GIP has been explored as a potential glucose-lowering drug based on its physiological role in augmenting glucosestimulated insulin secretion after meal ingestion. However, unlike GLP-1, GIP does not exert a sufficient insulinotropic effect in patients with type 2 diabetes, and has even been reported to increase glucagon levels [10]. Chemically modified GIP analogues have shown some glucose-lowering activity in hyperglycaemic rodent models, but they have not been tested in patients with type 2 diabetes. At the same time, GIP antagonists have been proposed to improve insulin sensitivity by preventing the development of obesity in rodents, but, similarly, such compounds have not yet been tested in patients with diabetes or obesity. In light of the partly opposing effects of GIP on insulin secretion and on fat deposition, we have doubts whether either agonists or antagonists at the GIP receptor will play a therapeutic role [11].

\section{Dual/triple peptide agonists or antagonists}

Glucose-lowering properties have been attributed not only to GLP-1, but also to GIP and other peptide hormones. Furthermore, glucagon receptor activation has led to improvements in obesity and insulin resistance in rodent models. While neither GIP nor glucagon seem to exert sufficient individual effects to justify their clinical use in the treatment of type 2 diabetes and obesity, the combination of these hormones with GLP-1 may represent a new treatment strategy in the near future. By generating hybrid peptides, various receptors can now be activated or blocked by a single compound. Oxyntomodulin is a physiological example of a peptide that activates both GLP1 and glucagon receptors. The synthesis of hybrid peptides allows the affinity of the drugs toward different receptors to be optimised. Triple agonists may target GLP-1, GIP and glucagon receptors [12]. From a theoretical standpoint, combination of these peptide actions sounds intriguing. Nevertheless, given the complexity of the individual hormone actions and the combined side effects of each peptide component, the challenge will be to fine-tune the structure of hybrid peptides in such a way that optimum weight loss and glycaemic control are achieved. A particular challenge will be the transition from pre-clinical to clinical studies, since there are likely to be species differences. The balance of affinities towards the different 
receptors may need to be adjusted to produce optimum results in humans.

\section{Other gut-derived targets for pharmacotherapy}

Although GIP and GLP-1 have been identified as the most important humoral mediators of the incretin effect, other gutderived factors are also involved in glucose and energy homoeostasis and may therefore qualify as future therapeutic targets [13] (Fig. 1). The L cell-derived hormone peptide YY (PYY) has a potent appetite-suppressing effect, thereby opening a potential avenue for the treatment of obesity and type 2 diabetes. In contrast, ghrelin, which is secreted from gastric epsilon cells, promotes hunger. Therefore, antagonising ghrelin may be a suitable strategy for body weight reduction. Other gut hormones, such as gastrin, secretin, cholecystokinin, vasoactive intestinal polypeptide (VIP) and pituitary adenylate cyclase-activating polypeptide (PACAP), stimulate insulin secretion at supraphysiological concentrations. On this basis, a therapeutic role in the treatment of type 2 diabetes appears theoretically possible [13]. More recently, high concentrations of bile acids have been associated with increased GLP-1 concentrations and glycaemic improvements, which might be mediated through nuclear farnesoid-receptor X (FXR) and the Gprotein-coupled bile acid-activated receptor (TGR5) on enteroendocrine L cells [14]. These receptors might also themselves be potential targets for treatment approaches aiming to enhance GLP-1 secretion. Finally, increasing evidence has linked changes in the intestinal microbiome with changes in insulin sensitivity and glucose metabolism. Future therapeutic strategies for type 2 diabetes may therefore also aim to modulate the bacterial composition in the gut (Fig. 1).

\section{Pharmacotherapy based on lessons from bariatric surgery}

The resolution of diabetes achieved by bariatric surgery in a large proportion of patients with type 2 diabetes has prompted some surgeons to propose such procedures as a favourable treatment strategy for patients with type 2 diabetes even in the absence of morbid obesity [15]. We do not believe that bariatric surgery will continue to develop into a widely used treatment strategy to treat the majority of patients 50 years from now, given the peri-interventional mortality and morbidity risks, malabsorptive problems and other complications. However, currently performed bariatric procedures may guide future research activities to develop conservative treatment strategies copying the hormonal environment created after, e.g. Roux-en-Y gastric bypass surgery, but without the inherent risks of major surgical procedures. To date, research interest has focused on the incretin hormones GIP and GLP-1. Exploring the mechanisms of diabetes resolution after bariatric surgery may help to identify additional factors that could potentially be employed for conservative treatment approaches. Those mechanisms may include changes in the secretion of gut hormones, gastrointestinal absorption and bile acid secretion, as well as the intestinal microbiome (see above). Furthermore, intestinal secretion of cytokines, such as fibroblast growth factors 19 and 21 , may mediate glycaemic improvements after bariatric surgery [14]. Using pharmacological approaches to mirror the surgery-induced changes in these and potentially additional factors by pharmacological approaches may lead to further improvements in glycaemia and weight regulation.

\section{Incretin-based therapies: dealing with safety concerns}

Long-term safety concerns related to the induction of tachycardia (GLP-1 receptor agonists only), a potential risk of pancreatitis, pancreatic cancer, thyroid cancer, colon cancer, cholecystitis and heart failure (in a trial with saxagliptin) have been expressed $[4,16]$. Nevertheless, data available at this point in time still indicate a favourable risk-benefit ratio.

\section{Future indications for incretin-based therapies}

At present, the application of incretin-based therapies is mainly restricted to the pharmacotherapy of type 2 diabetes, but various studies have already raised the prospect for a broader use of these drugs in other indications (Fig. 1). An obvious future indication for incretin-based therapies is the treatment of individuals with impaired glucose tolerance (IGT) or impaired fasting glucose (IFG) [17]. Treating individuals with obesity and impaired glucose metabolism with GLP-1 receptor agonists has already been reported to prevent the onset of diabetes, at least for a treatment period lasting 1 or 2 years [17]. Also, smaller-scale trials with DPP-4 inhibitors have suggested potential benefits in individuals with impaired glucose metabolism [18]. The main barrier to the pharmacological treatment of such individuals is probably the fact that IFG or IGT are not acknowledged as true disease entities, and are therefore not conventional indications for pharmacotherapy. In light of the somewhat arbitrary definitions of diabetes and IFG/IGT, such categorical thinking should be rethought in the future.

Women with a history of gestational diabetes, women with polycystic ovary syndrome, and other populations at high risk of developing type 2 diabetes may also benefit from preventative intervention with incretin-based drugs.

The incidence and prevalence of obesity has reached epidemic proportions. Recently, GLP-1 receptor agonists have been approved for the treatment of obesity in some parts of the world [19], and will likely be increasingly used for this indication in the future. Whether weight reductions achieved with the currently available doses and formulations of GLP-1 receptor agonists will justify lifelong treatment of obesity is an open question. The potential benefits of such treatment (long- 
term weight reduction, prevention of diabetes and diabetic complications, including cardiovascular events) need to be demonstrated in large clinical trials, but this earlier-stage population may provide unique chances to reveal benefits that cannot be shown at later stages, when the degree of tissue damage has advanced to an irreversible state. Another disease group that might benefit from treatment with incretin-based therapies is non-alcoholic fatty liver disease (NAFLD) or nonalcoholic steatohepatitis (NASH).

A number of recent studies have provided evidence that DPP-4 inhibitors and GLP-1 receptor agonists can improve glycaemic control as an adjunct to insulin therapy in patients with type 1 diabetes (for example, see [20]). Whether such combinations will still be used 50 years from now will also depend on whether there are improvements in other areas, such as immunotherapy or closed-loop insulin delivery systems.

Various cardiovascular outcome studies with incretinbased therapies are currently being conducted [21]. If these studies should reveal a cardiovascular benefit, even in the absence of major differences in glycaemia, we might see a broader use of incretin-based drugs in the treatment of coronary heart disease. However, the cardiovascular outcome trials that have been completed so far have failed to show cardioprotection with DPP-4 inhibitor treatment, which might be partly attributable to limitations of the study design, patient selection and trial duration. Whether this is also true for longer-term treatment or for GLP-1 analogues is not yet clear at this point in time.

Finally, neurodegeneration may evolve as a future indication for incretin-based therapies. Numerous studies have provided evidence for histological and functional improvements in rodent models of Alzheimer's, Parkinson's and other neurodegenerative diseases. Recently, improvements in memory and movement disorders have also been reported in patients with Parkinson's disease, thus revealing a therapeutic potential for the incretin-based therapies in this field [22].

\section{How can future research in the incretin field be further enhanced?}

Almost 30 years have passed since GLP-1 was first identified as an insulinotropic agent. Numerous physiological insights and therapeutic approaches have been derived from dedicated academic research supplemented with serendipity and a wideangled view on the clinical potential. However, many of the novel questions related to a wide array of biological effects of GLP-1 will have to be answered in future years. Fifty years from now, we hope to see more therapeutic approaches based on the properties of GLP-1 and related peptides, optimised to treat patients according to their individual needs, with broader indications extending into areas beyond classical glucose- lowering therapy. Given the many surprising results reported in the past, some unexpected findings might even boost this development further.

Duality of interest JJM has received consulting and speaker honoraria from AstraZeneca, Bristol-Myers Squibb, Eli Lilly \& Co., Merck Sharp \& Dohme GmbH, Novo Nordisk and Sanofi. He has received research support from Eli Lilly, Boehringer Ingelheim, MSD, Novo Nordisk, Novartis and Sanofi.

MAN has participated in advisory boards or was invited as speaker by Amylin Pharmaceuticals, AstraZeneca, Berlin Chemie, Boehringer Ingelheim, Bristol-Myers Squibb, Diartis Pharmaceuticals, Inc., Eli Lilly \& Co., GlaxoSmithKline, Hoffmann-La Roche Ltd, Intarcia Therapeutics, Inc., Janssen Global Services, MannKind Corp., Merck Sharp \& Dohme GmbH, Novo Nordisk, Novartis, Sanofi-Aventis, Takeda, Versartis, Wyeth Research. He has received research support from AstraZeneca, Berlin Chemie, Boehringer Ingelheim, Eli Lilly \& Co., GlaxoSmithKline, Merck Sharp \& Dohme, MetaCure, Inc., Novartis, Roche Pharma, Novo Nordisk and Tolerx, Inc.

Contribution statement Both authors were responsible for drafting the article and revising it critically for important intellectual content. Both authors approved the final version to be published.

\section{References}

1. Nauck M, Stöckmann F, Ebert R, Creutzfeldt W (1986) Reduced incretin effect in type 2 (non-insulin-dependent) diabetes. Diabetologia 29:46-54

2. Deacon CF, Danielsen P, Klarskov L, Olesen M, Holst JJ (2001) Dipeptidyl peptidase IV inhibition reduces the degradation and clearance of GIP and potentiates its insulinotropic and antihyperglycemic effects in anesthetized pigs. Diabetes 50: 1588-1597

3. Nauck MA, Kleine N, Ørskov C, Holst JJ, Willms B, Creutzfeldt W (1993) Normalization of fasting hyperglycaemia by exogenous glucagon-like peptide 1 (7-36 amide) in type 2 (non-insulin-dependent) diabetic patients. Diabetologia 36:741-744

4. Meier JJ (2012) GLP-1 receptor agonists for individualized treatment of type 2 diabetes mellitus. Nat Rev Endocrinol 8:728-742

5. Gough SC, Bode B, Woo V et al (2014) Efficacy and safety of a fixed-ratio combination of insulin degludec and liraglutide (IDegLira) compared with its components given alone: results of a phase 3, open-label, randomised, 26-week, treat-to-target trial in insulin-naive patients with type 2 diabetes. Lancet Diab Endocrinol 2:885-893

6. Nauck MA (2011) Incretin-based therapies for type 2 diabetes mellitus: properties, functions, and clinical implications. Am J Med 124:S3-S18

7. Nauck MA, Baranov O, Ritzel RA, Meier JJ (2013) Do current incretin mimetics exploit the full therapeutic potential inherent in GLP-1 receptor stimulation? Diabetologia 56:1878-1883

8. Tahrani AA, Bailey CJ, Del Prato S, Barnett AH (2011) Management of type 2 diabetes: new and future developments in treatment. Lancet 378:182-197

9. Madsbad S, Holst JJ (2014) GLP-1 as a mediator in the remission of type 2 diabetes after gastric bypass and sleeve gastrectomy surgery. Diabetes 63:3172-3174

10. Meier JJ, Nauck MA (2004) Clinical endocrinology and metabolism. Glucose-dependent insulinotropic polypeptide/gastric 
inhibitory polypeptide. Best Pract Res Clin Endocrinol Metab 18: 587-606

11. Meier JJ, Nauck MA (2004) GIP as a potential therapeutic agent? Horm Metab Res 36:859-866

12. Finan B, Yang B, Ottaway N et al (2015) A rationally designed monomeric peptide triagonist corrects obesity and diabetes in rodents. Nat Med 21:27-36

13. Nauck MA, Meier JJ (2008) Incretins and regulation of insulin secretion. In: Seino S, Bell GI (eds) Pancreatic beta cell in health and disease. Springer, Tokyo, pp 335-378

14. Madsbad S, Dirksen C, Holst JJ (2014) Mechanisms of changes in glucose metabolism and bodyweight after bariatric surgery. Lancet Diab Endocrinol 2:152-164

15. Mingrone G, Panunzi S, De Gaetano A et al (2012) Bariatric surgery versus conventional medical therapy for type 2 diabetes. $\mathrm{N}$ Engl J Med 366:1577-1585

16. Koehler JA, Baggio LL, Yusta B et al (2015) GLP-1R agonists promote normal and neoplastic intestinal growth through mechanisms requiring Fgf7. Cell Metab 21:379-391
17. Astrup A, Rossner S, Van Gaal L et al (2009) Effects of liraglutide in the treatment of obesity: a randomised, double-blind, placebocontrolled study. Lancet 374:1606-1616

18. Utzschneider KM, Tong J, Montgomery B et al (2008) The dipeptidyl peptidase-4 inhibitor vildagliptin improves beta-cell function and insulin sensitivity in subjects with impaired fasting glucose. Diabetes Care 31:108-113

19. Wadden TA, Hollander P, Klein S et al (2013) Weight maintenance and additional weight loss with liraglutide after low-calorie-dietinduced weight loss: the SCALE Maintenance randomized study. Int J Obes (Lond) 37:1443-1451

20. Kielgast U, Krarup T, Holst JJ, Madsbad S (2011) Four weeks of treatment with liraglutide reduces insulin dose without loss of glycemic control in type 1 diabetic patients with and without residual beta-cell function. Diabetes Care 34:1463-1468

21. Ussher JR, Drucker DJ (2014) Cardiovascular actions of incretinbased therapies. Circ Res 114:1788-1803

22. Aviles-Olmos I, Dickson J, Kefalopoulou Z et al (2013) Exenatide and the treatment of patients with Parkinson's disease. J Clin Invest 123:2730-2736 